\title{
Atemwegs- und Lungenerkrankungen in der Europäischen Landwirtschaft
}

Teil 1: Literaturübersicht

K. Radon

D. Nowak

\author{
Respiratory Diseases in European Farmers. Part 1: Literature Review
}

\section{Zusammenfassung}

Landwirtschaft ist nach wie vor weltweit ein bedeutender Wirtschaftssektor. Eine hohe Prävalenz von Atemwegserkrankungen bei Landwirten ist bekannt. Im Vordergrund stehen hierbei das allergische und nicht-allergische Asthma bronchiale, die chronische Bronchitis, die exogen-allergische Alveolitis sowie das Organic Dust Toxic Syndrome (ODTS). Aufgrund der Vielgestaltigkeit des landwirtschaftlichen Spektrums innerhalb Europas können sich die Expositionen und damit die Risikofaktoren für Atemwegserkrankungen stark unterscheiden. Während in der Getreideverarbeitung und in der Nutztierhaltung insbesondere die Exposition gegenüber organischen Stäuben und irritativen Gasen im Vordergrund steht, sind Beschäftigte in Gewächshäusern vorrangig gegenüber Pollen, Pilzsporen und Pestiziden exponiert. Bislang war das Wissen über die Prävalenz von Atemwegserkrankungen in den verschiedenen Zweigen der europäischen Landwirtschaft mangels epidemiologischer Untersuchungen unter Einsatz standardisierter Erhebungsinstrumente stark eingeschränkt. In diesem Zusammenhang fehlten auch zuverlässige Angaben über die für die Entwicklung von Atemwegserkrankungen relevanten Betriebscharakteristika und Expositionsparameter. Aus diesem Grund wurde die im zweiten Teil dieses Artikels vorgestellte europäische Landwirtschaftsstudie initiiert.

\section{Abstract}

Farming is still one of the most important economic sectors in the world. At the same time, a high prevalence of respiratory diseases in farmers is well known. Among these, allergic and non-allergic asthma, chronic bronchitis, hypersensitivity pneumonitis and organic dust toxic syndrome (ODTS) are of uppermost importance. Because of the large variety of agriculture across Europe exposure conditions and risk factors for airway diseases may vary largely. While exposure to organic dusts and irritants are most important in grain and animal production, workers in greenhouses are mainly exposed to pollen, fungi, as well as pesticides. Up to now, the knowledge about the prevalence of respiratory diseases in European farmers working in different agricultural sectors was limited. Furthermore, reliable data on farming characteristics as well as exposure patterns that might prone risk factors for respiratory diseases were missing. Therefore, the European Farmers' Project was initiated. The results of this study are given in the second part of this paper.

Institutsangaben

Institut und Poliklinik für Arbeits- und Umweltmedizin, Ludwig-Maximilians-Universität München

Korrespondenzadiresse

PD Dr. Katja Radon · Arbeitsgruppe Arbeits- und Umweltepidemiologie \& Net Teaching · Institut für Arbeitsund Umweltmedizin · Ziemssenstr. 1 80336 München · E-mail: kradon@arbeits.med.uni-muenchen.de

Eingereicht: 24. April 2003 · Nach Revision angenommen: 26. Juni 2003

Bibliografie

Pneumologie 2003; 57: 444-448 @ Georg Thieme Verlag Stuttgart · New York · ISSN 0934-8387 
Landwirtschaft ist die weltweit größte Wirtschaftsbranche. In vielen Ländern arbeiten mehr als 70\% der Erwerbstätigen in der Landwirtschaft. Innerhalb der Europäischen Union ist die Landund Forstwirtschaft nach wie vor ein wichtiger Wirtschaftssektor mit mehr als 7 Millionen Berufstätigen entsprechend 4,7\% aller Erwerbstätigen [20]. Unter Gesichtspunkten der öffentlichen Gesundheitspflege ist somit der Erhalt der Gesundheit von Landwirten $^{1}$ von hoher Bedeutung.

Die Arbeit in der Landwirtschaft wurde schon früh mit der Entstehung von Atemwegserkrankungen in Zusammenhang gebracht. Bereits 1555 wies Olaus Magnus auf die Gefährdung des Respirationstraktes durch die Arbeit in der Landwirtschaft hin [35]. Zahlreiche epidemiologische Untersuchungen bestätigten seither diese Assoziation [36]. In landwirtschaftlichen Betrieben findet sich in Abhängigkeit von der Betriebsart eine Vielzahl verschiedener gas- und staubförmiger Schadstoffe, die den Respirationstrakt des Landwirts schädigen können. Daraus resultierend stehen Erkrankungen des Atemtrakts auch bei den Berufskrankheiten im Bereich der landwirtschaftlichen Berufsgenossenschaften an zweiter Stelle nach von Tieren auf Menschen übertragbaren Erkrankungen [5]. Die Vermeidung dieser Erkrankungen des Respirationstrakt bei Landwirten stellt aufgrund der hohen Prävalenz der Erkrankungen in dieser Berufsgruppe sowie der großen Anzahl von Landwirten weltweit eine wichtige Aufgabe der präventiven Arbeitsmedizin mit großer Relevanz für die öffentliche Gesundheit dar.

\section{Besonderheiten der Berufsgruppe der Landwirte}

Die Berufsgruppe der Landwirte unterscheidet sich in verschiedenen Aspekten von anderen arbeitsmedizinischen Kollektiven. Der Anteil an Rauchern und Ex-Rauchern ist bei Landwirten gering [39]. In Deutschland sind bislang die meisten Berufstätigen in der Landwirtschaft Eigentümer der Betriebe bzw. deren Familienangehörige. Bei diesen spielt der „Healthy worker Effekt“, ein Selektionsphänomen, das in vielen beruflich exponierten Berufsgruppen zu einer Risikounterschätzung in Längs- und Querschnittsstudien führt [34], eine untergeordnete Rolle [42]. Weiterhin beginnen Landwirte ihre berufliche Tätigkeit auf dem Hof häufig bereits in der Kindheit und führen sie bis über das Rentenalter hinaus aus. Die ersten Kontakte zur Stallumgebung finden oft schon in den ersten Lebenswochen statt [57]. Da die meisten Landwirte auch auf dem landwirtschaftlichen Betrieb leben, ist die Exposition nicht auf eine definierte Arbeitszeit beschränkt. Besonders in kleinen Familienbetrieben besteht nur in geringem Maß Aufgabenteilung, so dass die Landwirte oftmals gegenüber einem breiten Spektrum von potenziellen Schadstoffen exponiert sind. Die Art der Aufgaben und damit der Expositionen unterliegt darüber hinaus einer starken saisonalen Variabilität.

\section{Atemwegserkrankungen in der Landwirtschaft}

Atemwegserkrankungen gehören $\mathrm{zu}$ den häufigsten Berufskrankheiten in der Landwirtschaft. Zu diesen zählen insbesondere die Berufskrankheiten 4301 und 4302 und - zu einem deutlich

\footnotetext{
${ }^{1}$ Der Begriff „Landwirt“ umfasst im Folgenden sowohl selbständige Landwirte als auch Angestellte auf landwirtschaftlichen Betrieben.
}

geringeren Prozentsatz - die exogen-allergische Alveolitis [5]. So wurden im Jahr 2000 insgesamt 590 Berufskrankheitenanzeigen aufgrund obstruktiver Atemwegserkrankungen bei den landwirtschaftlichen Berufsgenossenschaften gestellt, aber nur 126 mit dem Verdacht einer exogen-allergische Alveolitis [5]. Darüber hinaus wird diskutiert, ob das Organic Dust Toxic Syndrome (ODTS; Drescherfieber) in eine chronische Erkrankung übergehen kann [2]. Die wichtigsten Charakteristika dieser Erkrankungen bei Landwirten sind im Folgenden kurz zusammengefasst.

\section{Asthma}

Viele Fälle asthmatischer Erkrankungen bei Landwirten sind nicht IgE-vermittelt, sondern hängen mit einer chronischen Exposition gegenüber Irritantien zusammen. Eine solche Exposition kann vorbestehendes Asthma zwar verschlimmern, verursacht es aber nur selten. Manche Autoren bezeichnen derartige Krankheitsbilder als „Asthma-like-Syndrome“. Die Betroffenen berichten über giemende Atemgeräusche, Engegefühl in der Brust, trockenen Husten und/oder Kurzatmigkeit während der Arbeit. Teilweise gehen diese Symptome mit einem geringen Abfall der Einsekundenkapazität (meist < 10\%) über den Arbeitstag einher. Bei manchen Landwirten findet sich gleichzeitig ein vorübergehender Anstieg der unspezifischen bronchialen Empfindlichkeit [36].

\section{Chronisch-obstruktive Lungenerkrankungen}

Insbesondere die Zellwandbestandteile gramnegativer Bakterien und von Pilzen sind in der Lage, die Aktivität der Makrophagen zu steigern und können somit bei Landwirten mit erhöhter Exposition gegenüber organischen Stäuben eine neutrophile Entzündungsreaktion in den Atemwegen auslösen [55,58]. Diese Endotoxine und Glucane kommen insbesondere in Schweine- und Geflügelställen sowie in Getreidestäuben vor. Verschiedene Studien haben gezeigt, dass die anamnestische Angabe von Auswurf nicht mit der Atemwegsobstruktion des Landwirts korreliert. Es ist somit zu vermuten, dass die chronische, nicht-obstruktive Bronchitis bei Landwirten vielfach im Vordergrund steht [51].

\section{Exogen-allergische Alveolitis}

Die exogen-allergische Alveolitis (EAA; in der Landwirtschaft: „Farmerlunge“) ist eine Typ III und IV Allergie, die bei Landwirten primär durch die Bakterien Saccharopolyspora rectivirgula und Thermoactinomyces vulgaris sowie Schimmelpilze, insbesondere Aspergillus Spezies, verursacht wird. Es wird diskutiert, ob CoFaktoren wie beispielsweise Endotoxine oder $\beta-1,3$ Glucane benötigt werden, um die Erkrankung auszulösen [36].

\section{Organic Dust Toxic Syndrome}

Das mitunter nur schwer von der EAA zu unterscheidende Organic Dust Toxic Syndrome (ODTS; „Drescherfieber“) ist eine akut entzündliche Reaktion der Atemwege und Alveolen mit grippeähnlichen Allgemeinsymptomen. Charakteristisch ist das Auftreten von Symptomen 4-12 Stunden nach Exposition gegenüber hohen Konzentrationen an organischen Stäuben. Meist sind mehrere Exponierte von der Erkrankung gleichzeitig betroffen [36,52]. Typische Auslösesituationen sind das Ausmisten von Tierställen und die Arbeit mit schimmligem Getreide [54]. Es wird vermutet, dass Endotoxine maßgeblich an der Entstehung des ODTS beteiligt sind [58]. 
Risikofaktoren für Atemwegserkrankungen bei Landwirten im Pflanzenanbau

Bei der Verarbeitung von Getreide kommt es insbesondere zur Exposition gegenüber organischen Stäuben. In Getreidesilos können zudem höhere Konzentrationen an Gasen (Ammoniak, $\mathrm{CO}_{2}$, Stickoxide) vorkommen. Zum Auftreten von Atemwegssymptomen und -erkrankungen bei Beschäftigten im Getreideanbau liegen zahlreiche Untersuchungen aus verschiedenen Ländern vor $[2,36,38,53]$.

Diese weisen insbesondere auf ein erhöhtes Risiko für Irritationen der Nasenschleimhäute, ODTS und akute sowie chronische Bronchitiden hin, die wohl primär durch das in den Stäuben enthaltene Endotoxin hervorgerufen werden [2,53].

Größere epidemiologische Querschnittsstudien zu Atemwegserkrankungen bei Landwirten im Obst- und Gemüseanbau liegen primär aus Kalifornien vor [37]. Da die Böden in dieser ariden Region sehr silikatreich sind, wird von Schenker u. Mitarb. vermutet, dass bei den dortigen Landwirten im Pflanzenanbau das Risiko für Silikosen im Vordergrund steht [37]. In Europa spielt Silikatexposition im landwirtschaftlichen Bereich eine untergeordnete Rolle, systematische epidemiologische Untersuchungen größeren Umfangs zum Risiko für Atemwegserkrankungen bei im Obst- und Gemüseanbau Tätigen stehen noch aus.

Arbeit in Gewächshäusern kann den Atemtrakt durch Exposition gegenüber Pollen, Pilzsporen, Pestiziden und anderen Chemikalien beeinflussen $[3,4]$. Verschiedentlich wurde beschrieben, dass bei Beschäftigten in Gewächshäusern ${ }^{2}$ möglicherweise allergische Erkrankungen im Vordergrund stehen. Ein möglicher Zusammenhang zwischen der inhalativen Exposition gegenüber Carbamaten und Asthma bronchiale wurde dargestellt, diese Assoziation konnte für andere Pestizide nicht bestätigt werden [1]. Bisher liegen keine Untersuchungen zum Lungenfunktionsverlauf über den Arbeitstag bei Beschäftigten in Gewächshäusern vor. Überdies sind die Expositionsbedingungen in Gewächshäusern nur unzureichend charakterisiert.

\section{Risikofaktoren für Atemwegserkrankungen bei Landwirten in der Nutztierhaltung}

In der Tierproduktion kann es durch Güllegruben unter den Tierställen zu relevanten Expositionen gegenüber toxischen Gasen kommen [12]. Die wichtigsten Gase sind in diesem Zusammenhang Ammoniak, Methan und Schwefelwasserstoff. Insbesondere in Schweineställen wird der Grenzwert für Ammoniak, der je nach Land zwischen 20 und 40 ppm, in Deutschland bei 25ppm, liegt, häufig überschritten [14]. Darüber hinaus sind in Tierställen zahlreiche Quellen für organische Stäube zu finden. Die Hauptquelle dieser Stäube sind die Tiere selbst (z. B. Rinderepithelien, getrocknete Fäkalien) sowie Tierfutter (z. B. Getreide, Sojabohnen, Fischmehl) und Einstreu [28]. Die vielfältigen Expositionen in einem Tierstall sind in Abb. 1 beispielhaft für einen Schweinestall dargestellt.

\footnotetext{
2 Der Begriff „Beschäftigte in Gewächshäusern“ umfasst im Folgenden sowohl selbständige Landwirte als auch Angestellte mit Tätigkeiten in Gewächshäusern.
}

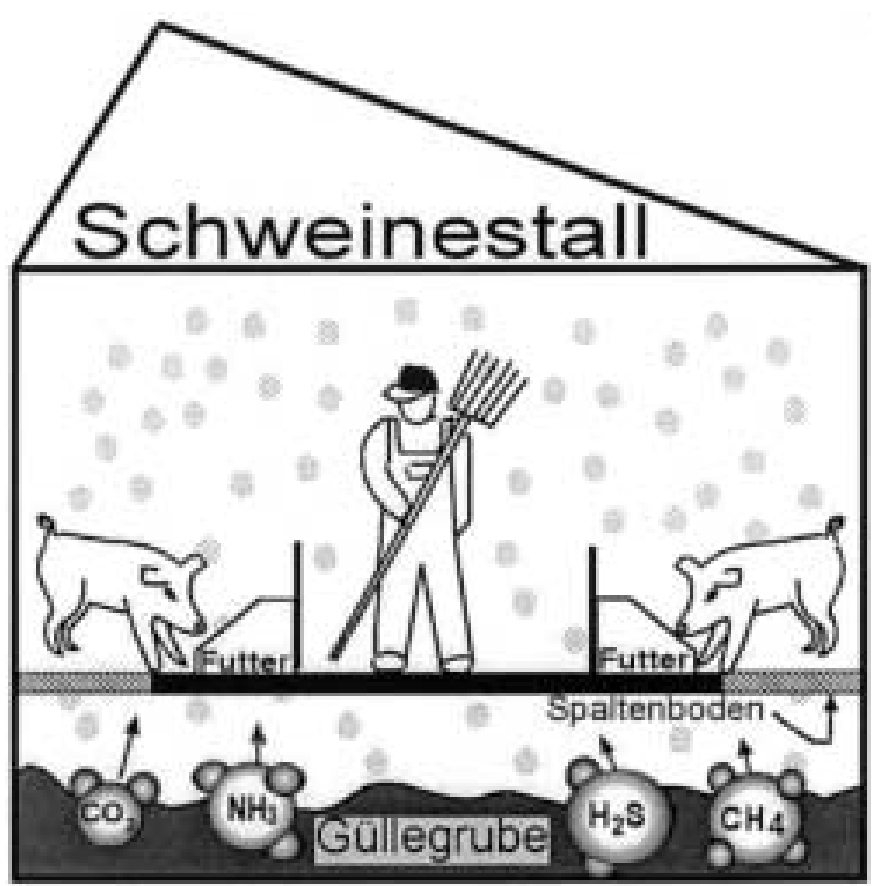

Abb. 1 Typische Emissionsquellen in einem Schweinestall

Daten zur Prävalenz von Atemwegserkrankungen in der Nutztierhaltung wurden bisher in zahlreichen Einzelstudien in verschiedenen Ländern erhoben [19,21,22,30,41]. Hierbei wurden in Abhängigkeit von den Erhebungsinstrumenten unterschiedliche Prävalenzen ermittelt. So wurde beispielsweise die Prävalenz von Asthma mit 2,6 [44] bis 11,8\% [26] angegeben. Viele Studien wurden nur an einer spezifischen Gruppe von Landwirten in der Tierproduktion erhoben $[6,13,24,26,56]$, die meisten hiervon bei Schweinezüchtern $[9-11,15-17,25,32,45-47,49]$. Aufgrund unterschiedlicher Methoden ist es nur unzureichend möglich, die Prävalenzen von Atemwegserkrankungen für Landwirte, die unterschiedliche Tierarten halten, zwischen den Studien zu vergleichen. Vergleichsdaten zur Prävalenz von Atemwegserkrankungen in der Allgemeinbevölkerung, die mit identischen Fragebogeninstrumenten erhoben wurden, liegen nur selten vor $[18,27]$.

Bei den bevölkerungsbezogenen Studien war die Anzahl der teilnehmenden Landwirte meist zu gering, um brauchbare Aussagen für unterschiedliche Arten der Tierhaltung zu erhalten $[23,29,43]$. Zur Prävalenz von Atemwegserkrankungen bei Landwirten in verschiedenen Ländern der Europäischen Union gibt es bisher keine standardisiert erhobenen Daten, die einen unmittelbaren Vergleich der Häufigkeit von Atemwegserkrankungen erlauben würden.

Nur wenige Querschnittsstudien erfassten die Häufigkeit von Atemwegserkrankungen bei Landwirten in der Geflügel- oder Schafhaltung. Die existierenden Untersuchungen zu Gesundheitsrisiken in der Geflügel- [7] und Schafhaltung [22] weisen auf eine hohe Prävalenz von Atemwegssymptomen hin. Expositionsmessungen wurden vereinzelt in Geflügelställen vorgenommen $[8,40]$. Über die Expositionsbedingungen bei Schafhaltern liegen bisher keine Studien vor. 
Für viele potenzielle Schadstoffe im landwirtschaftlichen Umfeld wie z. B. Endotoxine, Mykotoxine oder Allergene existieren keine wissenschaftlich validierten Expositionsgrenzwerte. So werden für Endotoxine Grenzwerte zwischen 5 und $200 \mathrm{ng} / \mathrm{m}^{3}$ diskutiert. Ebenso fehlen fundierte und zugleich praxisnahe Präventionskonzepte. Darüber hinaus ist bisher unklar, wie die Kombinationswirkungen von z.B. Tierepithelien, Desinfektionsmitteln und anderen chemischen Substanzen, Futterbestandteilen, Bakterien fäkaler Herkunft und Gasen wie beispielsweise Ammoniak in Schweineställen zu beurteilen sind. Aus ärztlicher Sicht sind Asthma und chronische Bronchitis dann vermeidbar, wenn die Risikofaktoren erkannt werden und geeignete Präventionsmaßnahmen ergriffen werden können. Während die Probenahme und Analytik für die Messung von Gasen und Stäuben standardisiert sind, ist bisher keine solche Standardisierung für die Bestimmung von Staubinhaltsstoffen erfolgt [36]. In landwirtschaftlichen Gebäuden (Tierställe, Gewächshäuser) wurde dem Zusammenhang zwischen Arbeitsplatzmerkmalen, wie beispielsweise Größe des Gebäudes, Einstreu, Futter oder Lüftungsparametern und der Exposition des Landwirtes wenig Aufmerksamkeit geschenkt. Die wenigen vorliegenden Studien zum Zusammenhang zwischen Arbeitsplatzmerkmalen und Atemwegserkrankungen wurden bei Schweinehaltern in den Niederlanden durchgeführt $[31,46,48,50]$. Hierbei wurden die Vermeidung des Einsatzes von Desinfektionsmitteln und Holzwolle sowie der Einsatz von mechanischen Ventilationssystemen als mögliche Präventionsmaßnahmen abgeleitet. Der Erfolg solcher Präventionsmaßnahmen wurde allerdings bislang in Interventionsstudien nicht überprüft.

Expositionsmessungen in den Stallungen und Gewächshäusern stellen nur eine Momentaufnahme der Exposition dar, die von Tag zu Tag variieren kann. Sie reflektieren daher oft nicht die für die Entstehung von Symptomen und Erkrankungen relevante Exposition in der Vergangenheit. Stallcharakteristika bleiben hingegen meist über einen längeren Zeitraum konstant und werden nur langsam durch Modernisierungsmaßnahmen verändert.

\section{Landwirtschaft in Europa}

Innerhalb Europas existiert ein breites landwirtschaftliches Spektrum. Während in Nordeuropa die Tierhaltung und der Getreideanbau im Vordergrund stehen, dominiert in Südeuropa der Pflanzenanbau. Zwischen verschiedenen Ländern variieren die Expositionsbedingungen bei Landwirten mit ähnlichen Aktivitäten aufgrund klimatischer Bedingungen oder landwirtschaftlicher Arbeitspraktiken. Ein Vergleich der Symptomprävalenzen von Landwirten in verschiedenen Produktionsbereichen, von Landwirten in verschiedenen Ländern Europas sowie zwischen Landwirten und der Allgemeinbevölkerung würde die Möglichkeit geben, Risikobereiche aufzudecken. Durch die zusätzliche Kenntnis der für die Entwicklung von Atemwegserkrankungen relevanten Betriebscharakteristika und Expositionsparameter in der Landwirtschaft könnten dann langfristig Präventionsmaßnahmen abgeleitet werden und somit ein wichtiger Beitrag zur Prävention von Atemwegserkrankungen in der Landwirtschaft geleistet werden.
Der nachfolgende Beitrag [33] stellt in diesem Zusammenhang die Ergebnisse einer europaweiten epidemiologischen Untersuchung in der Landwirtschaft vor. Darüber hinaus wurden unsere Ergebnisse der europäischen Untersuchungen mit Daten aus Kalifornien verglichen.

\section{Literatur}

${ }^{1}$ Alvarez E, Aurrekoetxea JJ, Santa ML et al. [Exposure to organophosphate insecticides among greenhouse workers in the Basque Country]. Med Clin (Barc) 1993; 101: 681-683

2 Baur X, Schneider WD. Nicht-allergische obstruktive Atemwegserkrankungen in der Landwirtschaft. Pneumologie 2000; 54: 80-91

${ }^{3}$ Bessot JC, Blaumeiser M, Kopferschmitt MC et al. [Occupational asthma in an agricultural setting]. Rev Mal Respir 1996; 13: 205-215

${ }^{4}$ Brauer M, Blair J, Vedal S. Effect of ambient ozone exposure on lung function in farm workers. Am J Respir Crit Care Med 1996; 154: 981-987

${ }^{5}$ Bundesverband der landwirtschaftlichen Berufsgenossenschaften. Zahl der anerkannten Berufskrankheiten bei den landwirtschaftlichen Berufsgenossenschaften im Jahr 2000. 2002

${ }^{6}$ Dalphin JC, Dubiez A, Monnet E et al. Prevalence of asthma and respiratory symptoms in dairy farmers in the French province of the Doubs. Am J Respir Crit Care Med 1998; 158: 1493 - 1498

${ }^{7}$ Danuser B. Respiratorische Gesundheitsrisiken bei Arbeiten mit Hühnern. Pneumologie 2000; 54: 37-42

${ }^{8}$ Danuser B, Wyss C, Hauser R et al. Lungenfunktion und Symptome bei Beschäftigten auf Hühnerfarmen. Soz Praventivmed 1988; 33: $286-291$

${ }^{9}$ Donham K, Haglind P, Peterson Y et al. Environmental and health studies of farm workers in Swedish swine confinement buildings. Br J Ind Med 1989; 46: $31-37$

${ }^{10}$ Donham KJ. Health effects from work in swine confinement buildings. Am J Ind Med 1990; 17: 17-25

${ }^{11}$ Donham KJ, Haglind P, Peterson Y et al. Environmental and health studies in swine confinement buildings. Am J Ind Med 1986; 10: 289-293

${ }^{12}$ Donham KJ, Knapp LW, Monson R et al. Acute toxic exposure to gases from liquid manure. J Occup Med 1982; 24: 142 - 145

${ }^{13}$ Donham KJ, Leistikow B, Merchant J et al. Assessment of U.S. poultry worker respiratory risks. Am J Ind Med 1990; 17: 73-74

${ }^{14}$ Donham KJ, Popendorf WJ. Ambient levels of selected gases inside swine confinement buildings. Am Ind Hyg Assoc J 1985; 46: 658 - 661

${ }^{15}$ Donham KJ, Reynolds SJ, Whitten P et al. Respiratory dysfunction in swine production facility workers: dose-response relationships of environmental exposures and pulmonary function. Am J Ind Med 1995; 27: $405-418$

${ }^{16}$ Donham KJ, Rubino M, Thedell TD et al. Potential health hazards to agricultural workers in swine confinement buildings. J Occup Med 1977; 19: $383-387$

${ }^{17}$ Donham KJ, Zavala DC, Merchant J. Acute effects of the work environment on pulmonary functions of swine confinement workers. Am J Ind Med 1984; 5: 367-375

18 Dosman JA, Graham BL, Hall D et al. Respiratory symptoms and pulmonary function in farmers. J Occup Med 1987; 29: 38-43

${ }^{19}$ Eduard W, Douwes J, Mehl R et al. Short term exposure to airborne microbial agents during farm work: exposure-response relations with eye and respiratory symptoms. Occup Environ Med 2001; 58: $113-118$

${ }^{20}$ Eurostat. Die Landwirtschaft in der Europäischen Union - Statistische und wirtschaftliche Informationen 1999. http://europa.eu.int/comm/ agriculture/agrista/table_de/fulltab.pdf, 1999

${ }^{21}$ Kimbell-Dunn M, Bradshaw L, Slater T et al. Asthma and allergy in New Zealand farmers. Am J Ind Med 1999; 35: 51 - 57

22 Kimbell-Dunn MR, Fishwick RD, Bradshaw L et al. Work-related respiratory symptoms in New Zealand farmers. Am J Ind Med 2001; 39: $292-300$

${ }^{23}$ Kogevinas M, Anto JM, Sunyer J et al. Occupational asthma in Europe and other industrialised areas: a population-based study. European Community Respiratory Health Survey Study Group. Lancet 1999; 353: $1750-1754$ 
${ }^{24}$ Kronqvist M, Johansson E, Pershagen G et al. Increasing prevalence of asthma over 12 years among dairy farmers on Gotland, Sweden: storage mites remain dominant allergens. Clin Exp Allergy 1999; 29: $35-41$

${ }^{25}$ Larsson K, Eklund A, Malmberg P et al. Alterations in bronchoalveolar lavage fluid but not in lung function and bronchial responsiveness in swine confinement workers. Chest 1992; 101: 767-774

${ }^{26}$ Malmberg P. Health effects of organic dust exposure in dairy farmers. Am J Ind Med 1990; 17: 7-15

${ }^{27}$ Manfreda J, Cheang M, Warren CP. Chronic respiratory disorders related to farming and exposure to grain dust in a rural adult community. Am J Ind Med 1989; 15: 7-19

${ }^{28}$ Merchant J, Donham K. Health risks from animal confinement units. In: Dosman J, Cockroft DW (Hrsg.): Principles of health and safety in agriculture. Florida: CRC Press, 1989: 58-61

${ }^{29}$ Monso E, Munoz-Rino F, Izquierdo J et al. Occupational asthma in the community: risk factors in a western Mediterranean population. Arch Environ Health 1998; 53: $93-98$

${ }^{30}$ Mustajbegovic J, Zuskin E, Schachter EN et al. Respiratory findings in livestock farmworkers. J Occup Environ Med 2001; 43: 576-584

${ }^{31}$ Preller L, Heederik D, Kromhout $\mathrm{H}$ et al. Determinants of dust and endotoxin exposure of pig farmers: development of a control strategy using empirical modelling. Ann Occup Hyg 1995; 39: 545-557

32 Quinn TJ, Donham KJ, Merchant JA et al. Peak flow as a measure of airway dysfunction in swine confinement operators. Chest 1995; 107: $1303-1308$

33 Radon K, Garz S, Riess A et al. Atemwegserkrankungen in der europäischen Landwirtschaft. Teil 2: Ergebnisse der euopäischen Landwirtschaftsstudie. Pneumologie 2003

${ }^{34}$ Radon K, Goldberg M, Becklake M. Healthy worker effect in cohort studies on chronic bronchitis. Scand J Work Environ Health 2002; 28: $328-332$

${ }^{35}$ Ramazzini B. De morbis artificum diatriba. Chicago IL: The University of Chicago Press, 1940

${ }^{36}$ Schenker M. Respiratory health hazards in agriculture. Am J Respir Crit Care Med 1998; 158: S1 - S76

37 Schenker M. Exposures and health effects from inorganic agricultural dusts. Environ Health Perspect 2000; 108 Suppl 4: 661 -664

${ }^{38}$ Schwartz DA, Thorne PS, Yagla SJ et al. The role of endotoxin in grain dust-induced lung disease. Am J Respir Crit Care Med 1995; 152: $603-608$

${ }^{39}$ Stellman SD, Boffetta P, Garfinkel L. Smoking habits of 800,000 American men and women in relation to their occupations. Am J Ind Med 1988; $13: 43-58$

${ }^{40}$ Takai HS, Pedersen S, Johsen JO et al. Concentrations and emissions of airborne dust in livestock buildings in Northern Europe. J Agri Eng Res 1998; 70: 59-77

41 Terho EO. Work-related respiratory disorders among Finnish farmers. Am J Ind Med 1990; 18: 269-272
42 Thelin A, Hoglund S. Change of occupation and retirement among Swedish farmers and farm workers in relation to those in other occupations. A study of "elimination" from farming during the period 1970 - 1988. Soc Sci Med 1994; 38: 147-151

${ }^{43}$ Toren K. Self reported rate of occupational asthma in Sweden 1990 - 1992. Occup Environ Med 1996; 53: 757 - 761

${ }^{44}$ U.S.Department of Health and Human Services. Work-related lung disease surveillance report. Cincinnati, $\mathrm{OH}$ : National Institute for Occupational Safety and Health,: 1999

${ }^{45}$ Vogelzang PF, Gulden JW van der, Cox AL. Hazards of inexperience in swine confinement work. Am J Ind Med 1993; 24: 261 - 263

${ }^{46}$ Vogelzang PF, Gulden JW van der, Folgering $\mathrm{H}$ et al. Longitudinal changes in bronchial responsiveness associated with swine confinement dust exposure. Chest 2000; 117: $1488-1495$

${ }^{47}$ Vogelzang PF, Gulden JW van der, Folgering $\mathrm{H}$ et al. Endotoxin exposure as a major determinant of lung function decline in pig farmers. Am J Respir Crit Care Med 1998; 157: 15-18

${ }^{48}$ Vogelzang PF, Gulden JW van der, Folgering $\mathrm{H}$ et al. Longitudinal changes in lung function associated with aspects of swine-confinement exposure. J Occup Environ Med 1998; 40: 1048-1052

${ }^{49}$ Vogelzang PF, Gulden JW van der, Folgering H. Organic dust toxic syndrome in swine confinement farming. Am J Ind Med 1999; 35: $332-334$

${ }^{50}$ Vogelzang PF, Gulden JW van der, Preller L. Bronchial hyperresponsiveness and exposure in pig farmers. Int Arch Occup Environ Health 1997; 70: $327-333$

${ }^{51}$ Essen S von. Bronchitis in agricultural workers. Sem Respir Med 1993; 14: $60-72$

52 Essen $\mathrm{S}$ von. Respiratory diseases related to work in agriculture. In: Langley M, Meggs W, Roberson G (Hrsg.): Agriculture, forestry and fisheries. Rockville: Government institutes, 1997: 353-384

${ }^{53}$ Essen $\mathrm{S}$ von. The role of endotoxin in grain dust exposure and airway obstruction. Curr Opin Pulm Med 1997; 3: 198-202

${ }^{54}$ Essen $\mathrm{S}$ von, Robbins RA, Thompson AB et al. Organic dust toxic syndrome: an acute febrile reaction to organic dust exposure distinct from hypersensitivity pneumonitis. J Toxicol Clin Toxicol 1990; 28: $389-420$

${ }^{55}$ Essen SG von, O'Neill DP, McGranaghan S et al. Neutrophilic respiratory tract inflammation and peripheral blood neutrophilia after grain sorghum dust extract challenge. Chest 1995; 108: 1425-1433

${ }^{56}$ Essen SG von, Scheppers LA, Robbins RA et al. Respiratory tract inflammation in swine confinement workers studied using induced sputum and exhaled nitric oxide. J Toxicol Clin Toxicol 1998; 36: 557-565

57 Mutius Evon, Braun-Fahrlander C, Schierl R et al. Exposure to endotoxin or other bacterial components might protect against the development of atopy. Clin Exp Allergy 2000; 30: 1230-1234

${ }^{58}$ Zejda JE, Dosman J. Respiratory disorders in agriculture. Tuber Lung Dis 1993; 74: 74-86 